\title{
Mucorales isolados do solo de mineração de cobre e produção de amilase e inulinase
}

\author{
André Luiz Cabral Monteiro Azevedo Santiago ${ }^{1}$ e Cristina Maria de Souza-Motta ${ }^{1,2}$
}

\author{
Recebido em 25/11/2003. Aceito em 16/03/2006
}

\begin{abstract}
RESUMO - (Mucorales isolados do solo de mineração de cobre e produção de amilase e inulinase). A presença de metais pesados no solo proporciona impacto sobre os microrganismos, vegetação e os processos funcionais do ecossistema. Visando comprovar que Mucorales são afetados pela extração de cobre na Mineradora Caraíba, Jaguarari, BA, espécies de Mucorales foram isoladas de amostras de solo coletadas nas épocas seca e chuvosa. Os Mucorales foram identificados e caracterizados quanto à capacidade de degradar amido e inulina. Utilizando os métodos de diluição e placa de solo, foram obtidos 46 isolados de Mucorales pertencentes a sete diferentes espécies. Absidia blakesleeana Lendner, A. cylindrospora Hagem, A. hialospora (Saito) Lendn., Cunninghamella elegans Lendner, Rhizopus microsporus V. Thieghen, R. oryzae Went. \& Prinsen Geerl. e Syncephalastrum racemosum (Cohn.) Schroet. foram isolados na época seca. A. blakesleeana e A. hialospora foram isoladas apenas na época chuvosa. Rhizopus oryzae apresentou maior número de isolados (30,43\%), seguido por A. blakesleeana (26,09\%) e C. elegans (21,74\%). O gênero que apresentou maior número de espécies foi Absidia seguido por Rhizopus. Entre as áreas, houve diferenças significativas no número de isolados de Mucorales, comprovando os efeitos negativos do impacto ambiental na abundância de Mucorales nos solos impactados. Todas as espécies degradaram o amido e apenas C. elegans degradou também inulina. Culturas de mesma espécie isoladas de áreas diferentes apresentaram o mesmo comportamento quanto à degradação de amido e inulina.
\end{abstract}

Palavras-chave: Mucorales, mineração do cobre, enzimas, semi-árido, caatinga

\begin{abstract}
Mucorales isolated from copper-mining soils and production of amylase and inulinase). The presence of heavy metals in soil causes impact on microorganisms, vegetation and functional processes in ecosystems. Aiming to prove that Mucorales are affected by copper extraction at the Caraíba mining site, Jaguarari, Bahia, species of Mucorales were isolated from samples collected in the dry and wet seasons. Mucorales were identified and characterized according to their capacity to degrade starch and inulin. Forty six isolates from seven different species of Mucorales were obtained by the serial-dilution and soil-plate methods. Absidia blakesleeana Lendner, A. cylindrospora Hagem, A. hialospora (Saito) Lendn., Cunninghamella elegans Lendner, Rhizopus microsporus V. Thieghen, $R$. oryzae Went. \& Prinsen Geerl. and Syncephalastrum racemosum (Cohn.) Schroet. were isolated in the dry season. Absidia blakesleeana and A. hialospora were isolated only in the wet season. Rhizopus oryzae had the greatest number of isolates (30.43\%) followed by A. blakesleeana (26.09\%) and C. elegans (21.74\%). The genus Absidia had the greatest number of species followed by Rhizopus. The number of isolates obtained from each sample site was significantly different, proving the negative effects of environmental impact on Mucorales abundance in the affected soils. All species were capable of degrading starch. However, C. elegans was the only species that also degraded inulin. Isolates of the same species from different areas showed the same behavior as regards capacity to degrade starch and inulin.
\end{abstract}

Key words: Mucorales, copper mining, enzymes, arid Brazil, caatinga

\section{Introdução}

O solo é considerado um dos principais reservatórios de populações de microrganismos e, dentre estes, encontram-se os fungos, constituindo um dos seres organotróficos responsáveis, primariamente, pela decomposição de compostos orgânicos (Paul \& Clark 1989). Neste habitat, os fungos são encontrados em comunidades variando de $10^{4}$ a $10^{6}$ organismos por grama de solo (Alexopoulos et al. 1996). A partir do solo são isolados fungos que podem ser utilizados para as mais diferentes finalidades, como produtores de antibióticos ou de enzimas, certos tipos de fermentadores ou degradadores de substâncias específicas (Cardoso et al. 1992).

Em solos de regiões de clima árido e semi-árido a composição de espécies de fungos é amplamente variável, não estando esses organismos distribuídos de maneira uniforme nos solos, mas concentrados em microhabitat que contém fontes orgânicas e condições abióticas favoráveis (Christensen 1981; Parkinson 1981; Skugins 1984).

1 Universidade Federal de Pernambuco, Departamento de Micologia, Centro de Ciências Biológicas, Av. Prof. Nelson Chaves, s/n, Cidade Universitária, 50670-420 Recife, PE, Brasil

2 Autor para correspondência: smotta@ufpe.br 
O distúrbio ambiental implica na desordem do ambiente normal dos fungos os quais podem adotar diferentes comportamentos diante da presença de elementos indesejáveis (Cooke \& Rayner 1984).

Os metais pesados e seus compostos podem interagir com os fungos de várias maneiras, dependendo das características do metal, organismo e do ambiente, estando mais intimamente associados à presença das indústrias e são conhecidos por causarem distúrbios nos microrganismos e na vegetação e por diminuírem a ciclagem de nutrientes do ecossistema. Metais como cobre $(\mathrm{Cu})$, zinco $(\mathrm{Zn})$ e cádmio $(\mathrm{Cd})$ podem ser tóxicos quando presentes em altas concentrações e em formas disponíveis, podendo ser acumulados pelos fungos (Valsecchi et al. 1995; Morley et al. 1996; Gaad et al. 2001).

Estudos iniciais acerca da distribuição de fungos em solos poluídos por cobre foram inicialmente realizados por Kendrick (1962), Lawrey (1977), Tatsuyama et al. (1974, 1977), Yamamoto et al. (1981) e Zibilske \& Wagner (1982) os quais reportaram os efeitos do cobre sobre o número de isolados de Rhizopus, Absidia, Mucor e Cunninghamella do solo. Nestes estudos, os autores constataram que solos poluídos por cobre apresentam uma diversidade de fungos reduzida, com maior ocorrência de espécies de Penicillium.

Muitas espécies de Zygomycetes são capazes de sintetizar produtos industriais como amilase, inulinase, pectinase, renina, ácidos orgânicos e outros metabólitos secundários (Nam 1993; Kim 1994; Ongen-Baysal et al. 1994; Cordeiro Neto et al. 1997). Dentre as enzimas de interesse industrial, a inulinase $(2,1-\beta$-D-fructanohidrolase EC 3.2.1.7) hidrolisa a inulina proporcionando um xarope rico em frutose $(95 \%)$, de grande importância na indústria alimentícia, por possuir maior poder adoçante, menores cariogenicidade e teor calórico (Bucke 1981; Vandame \& Derycke 1983; Bajpai \& Margaritis 1985; Fuchs et al. 1985). Na área médica, a inulinase é utilizada como ferramenta de diagnóstico em problemas renais (Kuehnle et al. 1992). Por outro lado, as amilases catalisam a hidrólise do amido e podem ser encontradas em plantas, animais e microrganismos, dentre esses, os fungos, sendo utilizadas na fabricação de medicamentos, papel, tecido, pão e na liquefação do amido (Lowe 1992).

Considerando-se o limitado conhecimento da diversidade de Mucorales em regiões de clima semiárido bem como o elevado potencial biotecnológico destes organismos em processos de biossorção e de produção enzimática, foram objetivos do presente trabalho: 1) isolar e identificar Mucorales do solo de uma área sob efeito de impacto ambiental causado por mineração de cobre: 2) comparar as espécies isoladas do solo das áreas impactadas ou não pela mineração e a influência da sazonalidade (período seco e chuvoso); 3 ) caracterizar qualitativamente as espécies de Mucorales isoladas quanto à capacidade de degradar amido e inulina, verificando se há diferença na atividade enzimática entre os isolados do solo provindos das diferentes áreas e 4) incorporar as espécies isoladas e caracterizadas ao acervo da Coleção de Culturas Micoteca URM do Departamento de Micologia do Centro de Ciências Biológicas da Universidade Federal de Pernambuco, pelos métodos de preservação em óleo mineral e água destilada esterilizada.

\section{Material e métodos}

Área de estudo - A mineração de cobre Caraíba está localizada em Pilar, Município de Jaguararí, BA (Latitude 9 51'43”S, longitude 3953'50”W). A região apresenta precipitação anual de $400 \mathrm{~mm}$, com chuvas ocorrendo principalmente nos meses de novembro a abril e temperatura média anual de $25^{\circ} \mathrm{C}$. A caatinga é a vegetação predominante e o solo na área estudada apresenta-se de dois tipos: areno-argiloso e argiloso, sendo o clima tropical semi-árido (Silva et al. 2001). As amostras de solo foram coletadas em agosto/1998 (época seca) e em fevereiro/1999 (época chuvosa). A mineradora foi subdividida em seis áreas (Silva et al. 2001), abaixo identificadas:

Área I - bacia de rejeito da mineradora, composta pelo pó da rocha da qual o minério é extraído.

Área II - arredores da mineradora (área de perímetro industrial).

Área III - local onde são depositados os restos de rochas com pouco ou nenhum minério coberto por fina camada de solo proveniente de outro local.

Área IV - área controle, sem impacto da mineradora (caatinga nativa).

Área V - interface entre a bacia de rejeito e a caatinga. Área com uma camada de rejeito de 20 a $50 \mathrm{~cm}$ sobre o solo natural.

Área VI - local onde é retirada a camada superficial do solo para compor a região onde são depositados os restos de rocha sem minério.

Em cada área foram coletadas sete amostras de solo (de 5 a $20 \mathrm{~cm}$ de profundidade), preferencialmente na rizosfera das plantas, sendo os pontos definidos aleatoriamente. Para cada ponto de coleta de solo foi 
considerado um raio de $2 \mathrm{~m}$ entre os pontos. As amostras coletadas em cada área foram misturadas obtendo uma amostra composta.

Análise química e granulométrica do solo - A análise do solo foi realizada pelo Laboratório de Solos da Embrapa Semi-Árido (Petrolina, PE), considerando a granulometria, $\mathrm{pH}$ e os seguintes elementos: fósforo $(\mathrm{P})$, alumínio $(\mathrm{Al})$, cobre $(\mathrm{Cu})$, ferro $(\mathrm{Fe})$, manganês (Mn) e zinco ( $\mathrm{Zn})$ (Tab. 1).

Isolamento e purificação dos Mucorales - Para o isolamento foi utilizado o método de placa de solo (Warkup 1950, modificado) em que $1 \mathrm{~g}$ do solo foi depositado em placa de Petri previamente esterilizada. Em seguida, foram adicionados $15 \mathrm{~mL}$ do meio de cultura Ágar Sabouraud (Lacaz et al. 2002) + Rosa de Bengala + cloranfenicol em cada placa e o método de diluição em série (Clark 1965, modificado) em que $25 \mathrm{~g}$ de solo foram suspensos em $225 \mathrm{~mL}$ de água destilada esterilizada $(1: 10 \mathrm{p} / \mathrm{v})$. Desta suspensão, $10 \mathrm{~mL}$ foram transferidos para $990 \mathrm{~mL}$ de água destilada esterilizada (1:1000), da qual $1 \mathrm{~mL}$ foi espalhado na superfície do meio Ágar Sabouraud + cloranfenicol contido em placas de Petri. O crescimento das colônias foi acompanhado por 72 horas à temperatura ambiente (TA) de $28 \pm 1{ }^{\circ} \mathrm{C}$. Para a purificação dos Mucorales, fragmentos das colônias foram transferidos separadamente para o meio Ágar Sabouraud + cloranfenicol contido em placa de Petri e, após confirmada pureza, transferidos para tubos de ensaio contendo Batata Dextrose Ágar - BDA (Lacaz et al. 2002). Durante o isolamento, foram realizadas três repetições para cada área.

Identificação dos Mucorales - As espécies foram identificadas pela observação das características macroscópicas (coloração, aspecto e diâmetro das colônias) e microscópicas (microestruturas), de acordo com Hesseltine \& Fennel (1995), Benny (1982), Schipper (1984), Schipper (1990) e Domsch et al. (1980).

Caracterização enzimática quanto à produção de amilase e inulinase - Fragmentos das culturas com sete dias de crescimento foram transferidos para o centro de placas de Petri contendo meio de cultura Ágar Czapek (Lacaz et al. 2002), modificado com os seguintes tratamentos: a) meio controle negativo (CN) sem fonte de carbono $\left(\mathrm{NaNO}_{3} 3 \mathrm{~g}, \mathrm{MgSO}_{4} 0,5 \mathrm{~g}\right.$, $\mathrm{FeSO}_{4}+7 \mathrm{H}_{2} \mathrm{O} 0,01 \mathrm{~g}, \mathrm{~K}_{2} \mathrm{HPO}_{4} 1 \mathrm{~g}$, Ágar $16 \mathrm{~g}$ e água destilada $1.000 \mathrm{~mL}$ ); b) controle positivo $(\mathrm{CP})$ (meio $\mathrm{CN}$ adicionado de $10 \mathrm{~g}$ de glicose); c) meio I (meio $\mathrm{CN}$ adicionado de $10 \mathrm{~g}$ de inulina) e d) meio A ( meio $\mathrm{CN}$ adicionado de $10 \mathrm{~g}$ de amido). Após 5 dias à TA, a taxa crescimento dos Mucorales inoculados nos meios I e A foi comparada com a taxa observada nas placas com o meio $\mathrm{CN}$ e $\mathrm{CP}$ utilizando-se a fórmula $\mathrm{Tx}=\mathrm{Ix}$ $100 / \mathrm{G}$ e $\mathrm{Tx}=\mathrm{A} \times 100 / \mathrm{G}$, onde I e A representam o diâmetro da colônia respectiva nos meios I e A, e G o diâmetro da colônia no meio $\mathrm{CP}$ cujo crescimento foi considerado como sendo 100\% (Cordeiro Neto et al. 1997).

Estocagem dos Mucorales na Coleção de Culturas Micoteca URM - As espécies de Mucorales isoladas e caracterizadas foram estocadas em óleo mineral segundo Sherf (1943) e em água destilada esterilizada segundo Castellani (1939), na Coleção de Culturas Micoteca URM do Departamento de Micologia do Centro de Ciências Biológicas da Universidade Federal de Pernambuco. Os números de registro das espécies estocadas na Micoteca URM são: Absidia blakesleeana 4268, A. cylindrospora 4269 A. hialospora 4270, Cunninghamella elegans 4271,

Tabela 1. Análise química e granulométrica do solo das áreas da Mineradora Caraíba (BA). I = Bacia de rejeito; II = perímetro industrial; III = local onde são depositados os restos de rocha com pouco minério; IV = caatinga; V = interface entre a caatinga e a bacia de rejeito; $\mathrm{VI}=$ local onde foi retirada a camada superficial do solo.

\begin{tabular}{|c|c|c|c|c|c|c|c|c|c|c|}
\hline \multirow[t]{2}{*}{ Áreas } & \multirow[t]{2}{*}{$\mathrm{P}^{*}$} & \multirow[t]{2}{*}{$\mathrm{Cu}$} & \multirow{2}{*}{$\frac{\mathrm{Mn}}{\left(\mathrm{mg} / \mathrm{dm}^{3}\right)}$} & \multirow[t]{2}{*}{$\mathrm{Zn}$} & \multirow[t]{2}{*}{$\mathrm{Fe}$} & \multirow{2}{*}{$\begin{array}{c}\text { Al } \\
\text { Meq/100g } \\
\text { de solo }\end{array}$} & \multirow{2}{*}{$\begin{array}{l}\mathrm{pH} \\
\mathrm{H}_{2} \mathrm{O} \\
1: 2,5\end{array}$} & \multicolumn{3}{|c|}{ Granulometria } \\
\hline & & & & & & & & Areia & $\begin{array}{l}\text { Silte } \\
(\%)\end{array}$ & Argila \\
\hline I & 266 & 433,4 & 53,4 & 5,7 & 434,4 & 0,00 & 8,2 & 93 & 3 & 4 \\
\hline II & 44 & 71,9 & 33,4 & 2,0 & 31,8 & 0,04 & 6,9 & 60 & 24 & 16 \\
\hline III & 167 & 606,7 & 44,0 & 5,2 & 168,1 & 0,01 & 7,3 & 69 & 21 & 10 \\
\hline IV & 141 & 2,7 & 46,4 & 2,2 & 33,6 & 0,05 & 6,2 & 62 & 24 & 14 \\
\hline $\mathrm{V}$ & 179 & 445,4 & 47,8 & 5,0 & 337,1 & 0,00 & 7,8 & 61 & 35 & 4 \\
\hline VI & 95 & 30,4 & 48,6 & 1,6 & 52,4 & 0,17 & 7,1 & 65 & 19 & 16 \\
\hline
\end{tabular}

$*=$ Mehlich I. 
Rhizopus microsporus 4272, R. oryzae 4274, Syncephalastrum racemosum 4275.

Análise estatística - A verificação de uma possível distribuição diferencial no número de espécies de Mucorales, bem como de isolados dos mesmos nas diferentes áreas, foi realizada utilizando-se o teste de Mann-Withney (Berquó et al. 1981) e da diferença de proporções (Fleiss 1981), respectivamente. A possível influência da sazonalidade na distribuição dos isolados de Mucorales foi verificada pela Modelagem de Poisson (Bailey \& Gratell 1995).

\section{Resultados e discussão}

Espécies de Mucorales isoladas - Foram obtidos 46 isolados de Mucorales, sendo 12 na época seca e 34 na chuvosa, distribuídos em sete espécies: Absidia blakesleeana Lendner (12 isolados), A. cylindrospora Hagem (dois), A. hialospora (Saito) Lendn. (três) Cunninghamella elegans Lendner (dez), Rhizopus microsporus V. Thiegen (dois), R. oryzae Went. \& Prinsen Geerl. (14) e Syncephalastrum racemosum (Cohn.) Schroet. (três). O gênero que apresentou maior número de espécies foi Absidia (três espécies) seguido por Rhizopus (duas espécies) (Tab. 2). Rhizopus oryzae apresentou maior número de isolados $(30,43 \%)$ seguido por $A$. blakesleeana $(26,09 \%)$ e $C$. elegans (21,74\%). Absidia, Cunninghamella e Rhizopus foram também isolados por Zibilske \& Wagner (1982) e Yamamoto et al. (1985) de solos poluídos com cobre, corroborando os dados aqui obtidos.

$\mathrm{Na}$ área IV, considerada como área controle, pôde-se isolar um maior número de espécies e isolados de Mucorales, tendo em vista que esta área não sofreu impacto da mineração e apresenta, principalmente, baixos teores de cobre e ferro e pH 6,2 (Tab. 1).

As áreas II, III e VI sofreram impacto menos expressivo do que o observado para as áreas I e V, provavelmente pelos altos teores de $\mathrm{Cu}, \mathrm{Fe}$ e $\mathrm{P}$ e pelo $\mathrm{pH}$ alto $(7,8$ a 8,2$)$ das duas últimas. Aárea III, mesmo apresentando a maior concentração de $\mathrm{Cu}$, não estava totalmente desprovida destes fungos, o que ressalta o efeito limitante do $\mathrm{Fe}$ e $\mathrm{P}$, em combinação com o $\mathrm{Cu}$, na presença de Mucorales nessas áreas. (Tab. 1, 2). A redução do número de isolados de Absidia, Cunninghamella e Mucor pela elevação da concentração de $\mathrm{Cu}$ no solo foi citada anteriormente por Yamamoto et al. (1985). Silva et al. (2001), verificaram baixa ocorrência de fungos micorrízicos arbusculares nessas áreas, corroborando com o observado nesse trabalho. Segundo Souza \& Silva (1996), a retirada da vegetação natural, a intensa movimentação do solo e o acréscimo de considerável volume de rejeitos podem contribuir para o distúrbio do local. Este fato foi verificado principalmente para as áreas I, bacia de rejeito da mineradora, composta pelo pó da rocha da qual o minério é extraído, e V, interface entre a bacia de rejeito e a caatinga, com uma camada de rejeito de 20 a $50 \mathrm{~cm}$ sobre o solo natural.

A presença de Rhizopus oryzae em praticamente todas as áreas, nas duas estações do ano, sugere que a mesma seja a mais resistente às condições de impacto ambiental causado pela mineradora. Segundo Zibilske \& Wagner (1982) a presença de espécies de Rhizopus nos solos é elevada com o aumento da concentração de cobre. A utilização de R. oryzae em processos de biossorção de Cu e/ou Fe foi citada por Sag \& Kutsal (1996), Huang \& Huang (1996), Sag \& Kutsal (1998),

Tabela 2. Número de isolados das espécies de Mucorales obtidos do solo das áreas da Mineradora Caraíba (BA) durante as épocas seca e chuvosa. I, II, III, IV, V, VI = Áreas da Mineradora Caraíba (BA); S = Época seca; C = Época chuvosa.

\begin{tabular}{|c|c|c|c|c|c|c|c|c|c|c|c|c|c|}
\hline \multirow[t]{3}{*}{ Espécies } & \multicolumn{12}{|c|}{ Áreas } & \multirow{3}{*}{$\begin{array}{l}\text { Total de } \\
\text { isolados }\end{array}$} \\
\hline & \multicolumn{2}{|c|}{ I } & \multicolumn{2}{|c|}{ II } & \multicolumn{2}{|c|}{ III } & \multicolumn{2}{|c|}{ IV } & \multicolumn{2}{|c|}{ V } & \multicolumn{2}{|c|}{ VI } & \\
\hline & $\mathrm{S}$ & $\overline{\mathrm{C}}$ & $\mathrm{S}$ & $\mathrm{C}$ & $\mathrm{S}$ & $\mathrm{C}$ & $\mathrm{S}$ & $\overline{\mathrm{C}}$ & $\mathrm{S}$ & $\mathrm{C}$ & $\bar{S}$ & $\overline{\mathrm{C}}$ & \\
\hline Absidia blakesleeana Lendner & 0 & 0 & 0 & 2 & 0 & 0 & 0 & 7 & 0 & 0 & 0 & 3 & 12 \\
\hline A. cylindrospora Hagem & 0 & 0 & 0 & 0 & 0 & 0 & 1 & 0 & 0 & 0 & 0 & 1 & 2 \\
\hline A. hialospora (Saito) Lendn. & 0 & 0 & 0 & 0 & 0 & 0 & 0 & 3 & 0 & 0 & 0 & 0 & 3 \\
\hline Cunninghamella elegans Lendner & 0 & 0 & 0 & 0 & 0 & 0 & 0 & 8 & 0 & 0 & 1 & 1 & 10 \\
\hline Rhizopus microsporus V. Thieghen & 0 & 0 & 1 & 0 & 0 & 0 & 0 & 1 & 0 & 0 & 0 & 0 & 2 \\
\hline R. oryzae Went. \& Prinsen Geerl. & 0 & 0 & 2 & 1 & 2 & 5 & 2 & 1 & 0 & 0 & 1 & 0 & 14 \\
\hline Syncephalastrum racemosum (Cohn.) Schroet & 0 & 0 & 0 & 0 & 0 & 1 & 0 & 0 & 0 & 0 & 2 & 0 & 3 \\
\hline Total & 0 & 0 & 3 & 3 & 2 & 6 & 3 & 20 & 0 & 0 & 4 & 5 & 46 \\
\hline
\end{tabular}


Sag \& Kutsal (2000), Sag et al. (2000), Gupta et al. (2000), Sag et al. (2001) e Gaad et al. (2001), confirmando a tolerância dessa espécie a esses metais e corroborando os resultados deste trabalho. Volesky (1994) refere-se também às espécies de Absidia como eficientes em processos de biossorção de metais, sendo A. blakesleeana encontrada nas condições deste estudo com 12 isolados, ocorrendo, principalmente, no período chuvoso (Tab. 2).

A análise das diferenças do número de isolados de Mucorales entre as áreas da mineradora evidenciou diferenças estatísticas entre as proporções das áreas II e IV $(\chi 2=12,8911$, g.l. $=1, \mathrm{p}=0,0003)$, III e IV $(\chi 2=9,5357$, g.l. $=1, \mathrm{p}=0,002)$ e IV e VI $(\chi 2=$ $8,0979$, g.1. $=1, \mathrm{p}=0,0044)$, podendo-se afirmar que a área IV difere das demais em relação ao número de isolados. Não foram verificadas diferenças significativas no número de espécies de Mucorales entre as áreas da mineradora e o controle $(\mathrm{p}=0,317)$, indicando que o impacto da mineração influenciou na abundância das mesmas, mas não na diversidade. A influência da sazonalidade no número de isolados de Mucorales não foi evidenciada pela Modelagem de Poisson $(p=0,08)$, sendo os dados obtidos insuficientes para a constatação da influência da época do ano na diversidade de Mucorales nas áreas. Entretanto, Cardoso et al. (1992) ressaltam que em épocas de maior umidade há uma maior proporção de representantes de fungos por área de solo.

Maiores índices de similaridade de espécies, nas duas épocas do ano, ocorreram entre as áreas IV e VI; IV e II, com $72,72 \%$ e $66,66 \%$, respectivamente (Tab. 3). Esses resultados coincidem com os baixos teores de $\mathrm{Cu}$ e Fe nas áreas II e VI (Tab. 1), indicando que esses elementos afetam de forma marcante a ocorrência das espécies de Mucorales.

Caracterização enzimática dos isolados de Mucorales - Foram consideradas positivas as amostras

Tabela 3. Coeficiente de similaridade de Sorensen (Ss) entre as espécies de Mucorales obtidas do solo das áreas da Mineradora Caraíba (BA) durante as épocas seca e chuvosa.

\begin{tabular}{lcccccc}
\hline Áreas & I & II & III & IV & V & VI \\
\hline I & - & - & - & - & & - \\
II & 0 & - & - & - & - & - \\
III & 0 & $40 \%$ & - & - & - & - \\
IV & 0 & $66,66 \%$ & $25 \%$ & - & - & - \\
V & 0 & 0 & 0 & 0 & - & - \\
VI & 0 & $50 \%$ & $57,14 \%$ & $72,72 \%$ & 0 & - \\
\hline
\end{tabular}

que apresentaram, nos meios com amido e inulina, crescimento igual ou superior ao observado no meio de controle positivo (meio com glicose como fonte de carbono). Todas as espécies consideradas positivas para a produção de amilase e inulinase obtiveram taxa de crescimento de $100 \%$.

Todas as espécies isoladas nas duas épocas do ano hidrolisaram o amido. Apenas Cunninghamella elegans foi capaz de hidrolisar, também, a inulina. Kochhar et al. (1999) e Otha et al. (2002) constataram a produção de inulinase por $R$. oryzae e Rhizopus sp., respectivamente. Cordeiro Neto et al. (1997) observaram atividade inulinásica em Absidia cylindrospora isolada da rizosfera de Asteraceae do cerrado, embora não tenham constatado o mesmo para Cunninghamella elegans oriunda do mesmo substrato, contrariando os dados citados neste trabalho.

Os resultados referentes à atividade amilásica dos isolados de Rhizopus e Absidia aqui descritos são apoiados pelos de Chaurasia (1992), Amadioha (1998), Mitchell et al. (1988) e El-Zalaki \& Hamza (2003). No entanto, Stamford et al. (1998) não constataram atividade amilolítica em Rhizopus sp. isolado do Jacatupé (Pachyrhizus erosus L. Urban). Os dados deste trabalho indicam que culturas de mesma espécie isoladas de áreas diferentes apresentaram o mesmo comportamento quanto à degradação de amido e inulina.

É preciso salientar que são escassos os trabalhos referentes à Mucorales produtores de amilase e inulinase, estando nesse artigo, provavelmente, primeiros relatos de produção de inulinase por C. elegans e de amilase por A. cylindrospora, $A$. hialospora e $S$. racemosum.

O presente trabalho contribui para a ampliação do conhecimento da diversidade de Mucorales em solos de regiões de clima semi-árido, assim como em áreas impactadas pela mineração, tendo em vista a carência de informações sobre a distribuição desses organismos nessas regiões e o elevado potencial biotecnológico dos mesmos, principalmente no que se refere à produção de amilase e inulinase. Pesquisas que visem o desenvolvimento de novas técnicas e processos de otimização da produção dessas enzimas devem ser incentivadas, considerando-se sua a ampla atuação na biotecnologia e o elevado valor de mercado das mesmas. Além de $R$. oryzae, freqüentemente utilizado em processos de biossorção, $S$. racemosum deve ser utilizado em pesquisas envolvendo processos de biossorção de cobre e ferro, visto que essas espécies foram encontradas em áreas com altos teores desses metais. 


\section{Agradecimentos}

Às Professoras Dra. Maria Auxiliadora de Queiroz Cavalcanti, do Departamento de Micologia da Universidade Federal de Pernambuco, e a Dra. Sandra Farto Botelho Trufem, pela colaboração durante a identificação das espécies isoladas; ao Gladstone Alvez da Silva, pela coleta do solo da região da mineradora; ao CNPq, pelo apoio financeiro na Bolsa de Iniciação Científica.

\section{Referências bibliográficas}

Alexopoulos, C.J.; Mims, C.W. \& Blackwell, M. 1996. Introductory Mycology. New York, John Wiley \& Sons, Inc.

Amadioha, A.C. 1998. Effect of cultural conditions on the growth and amylolytic enzyme production by Rhizopus oryzae. Acta Phytopathologica Hungarica 33: 115-121.

Bailey, T.C. \& Gratell, A.C. 1995. Interactive Spatial Data Analysis. Longman Scientific \& Technical, Essex.

Bajpai, P. \& Margaritis, A. 1985. Immobilization of Kluyveromyces marxianus cells containing inulinase activity in open pore gelatin matrix: 2. Application for high fructose syrup production. Enzyme and Microbial Technology 7: 459-469.

Benny, G.L. 1982. Zygomycetes. Pp. 184-195. In: G.L. Benny (ed.). Synopsis and Classification of Living Organisms. New York, McGraw - Hill Book Co. Inc.

Berquó, E.S.; Souza, J.M.P. \& Gotlieb, S.L.D. 1981. Bioestatística. Pedagógica e Universitária, São Paulo.

Bucke, C. 1981. Enzyme in fructose manufacture. Pp. 51-72. In: G.G. Birch; N. Blakebrough \& K.J. Parker (eds.). Enzyme and Food Processing. London, Applied Science Publishers Ltd.

Cardoso, E.J.B.N.; Tsai, S.M. \& Neves, M.C.P. 1992. Microbiologia do Solo. Campinas, Sociedade Brasileira de Ciência do Solo.

Castellani, A. 1939. Viability of some pathogenic fungi in distilled water. Journal of Tropical Medicine and Hygiene 42: 225-226.

Chaurasia, S.C. 1992. Effect of fungicides on the production of amylase by Rhizopus oryzae. Hindustan Antibiotics Bulletin 34: 85-90.

Christensen, M. 1981. Species diversity and dominance in fungal communities. Pp. 201-232. In: D.T. Wicklow \& G.C. Carrol (eds.). The fungal community. New York, Marcel Dekker.

Clark, F.E. 1965. Agar-plate method for total microbial count. Pp. 1460-1466. In: C.A. Black; D. Evans; J.L. White; L.E. Ensminger; F.E. Clark \& R.C. Dinauer (eds.). Methods of soil analysis, Part 2. Chemical and microbiological properties. New York, Madson Inc.

Cooke, R.C. \& Rayner, A.D.M. 1984. Ecology of Saprophytic Fungi. London, Longman.
Cordeiro Neto, F.; Perssoni, R.A.B. \& Figueiredo-Ribeiro, R.C.L. 1997. Fungos produtores de inulinases isolados da rizosfera de Asteraceae herbácea do cerrado (MojiGuaçu, SP, Brasil). Revista Brasileira de Ciência do Solo 21: 149-153.

Domsch, K.H.; Gams, W. \& Anderson, T.H. 1980. Compendium of soil fungi. London, Academic Press.

El-Zalaki, E.M. \& Hamza, M.A. 2003. Edible mushrooms as producers of amylases. Food Chemistry 4: 203-211.

Fleiss, J.L. 1981. Statistical methods for rates and proportions. New York, Wiley.

Fuchs, A.; Brujin, J.M. \& Niedeveld, C.J. 1985. Bacteria and yeast as possible candidates for production of inulinases and levanases. Antonie Van Leewenhoek 51: 333-351.

Gaad, M.G.; Ramsay, L.; Crawford, J.W. \& Ritz, K. 2001. Nutritional influence on fungal colony growth and biomass distribution in response to toxic metals. FEMS Microbiology Letters 204: 301-316.

Gupta, R.; Ahuja, P.; Khan, S.; Saxena, R.K. \& Mohapatra, H. 2000. Microbial biosorbents: meeting challenges of heavy metal pollution in aqueous solutions. Current Science 78: 967-973.

Hesseltine, C.W. \& Fennel, D.I. 1995.The genus Circinella. Mycologia 7: 193-211.

Huang, C. \& Huang, P.C. 1996. Application of Aspergillus oryzae and Rhizopus oryzae for $\mathrm{Cu}$ (II) removal. Water Research 30: 1985-1990.

Kendrick, W.B. 1962. Soil fungi of copper swamp. Canadian Journal of Microbiology 8: 639-647.

Kim, M.K. 1994. Thermal stability of acidic inulinase from Scytalidium acidophilum. Biotechnology Letters 16(9): 965-966.

Kochhar, A.; Gupta, A.K. \& Kaur, N. 1999. Purification and immobilization of inulinase from Aspergillus candidus for producing fructose. Journal of Science Food Agricultural 79: 554-594.

Kuehnle, H.F.; Von Dahl, K. \& Schmidt, F.H. 1992. Fully enzymatic inulin determination in small volume samples without deproteinisation. Nephron 62: 104-107.

Lacaz, C.S.; Porto, E.; Martins, J.E.C.M.; Heis-Vaccari, E.M. \& Melo, N.T. 2002. Tratado de micologia médica. Lacaz. $9^{a}$ ed., São Paulo, Sarvier.

Lawrey, J.D. 1977. Soil fungal populations and respiration habitats influenced by coal strip-mining. Environmental Pollution 14: 195-205.

Lowe, D.A. 1992. Fungal enzymes. Pp. 681-706. In: D.K. Arora; R.P. Elander \& K.G. Mukergi (eds.). Handbook of Applied Mycology. Fungal Biotechnology. v.4, New York, Marcel Dekker Inc.

Mitchell, D.A.; Doelle, H.W. \& Greenfield, P.F. 1988. Agar plate growth studies of Rhizopus oligosporus and Aspergillus oryzae to determine their stability for solid-state fermentation. Applied Microbiology and Biotechnology 28: 598-602.

Morley, G.F.; Sayer, J.A.; Wilkinson, S.J.; Gharieb, M.M. \& Gadd, G.M. 1996. Fungal sequestration, solubilization and transformation of toxic metals. Pp. 235-256. In: J.C. Frankland; N. Magan \& G.M. Gadd (eds.). Fungi and Environmental Change. Cambridge, Cambridge University Press. 
Nam, S.W. 1993. Secretion and localization of invertase and inulinase in recombinant Saccharomyces cervisae. Biotechnology Letters 15(10): 1049-1054.

Ongen-Baysal, G. \& Sukan, S.S. 1994. Production and properties of inulinase from Aspergillus niger. Biotechnology Letters 16: 275-280.

Otha, K.; Suetsugu, N. \& Nakamura, T. 2002. Purification and properties of an extracellular inulinase from Rhizopus sp. strain TN-96. Journal of Bioscience and Bioengineering 94: 98-80.

Parkinson, D. 1981. Ecology of soil fungi. Pp. 227-294. In: G.T. Cole \& B. Kendrick (eds.). Biology of conidial fungi. New York, Academic press.

Paul, E.A. \& Clark, F.E. 1989. Soil Microbiology and Biochemistry. San Diego, Academic Press.

Sag, Y.; Ataçoglu, I. \& Kutsal, T. 2000. Equilibrium parameters for the single and multicomponent biosorption of $\mathrm{Cr}$ (VI) and Fe (III) ions on R. arrhizus in a packed column. Hydrometallurgy 55: 165-179.

Sag, Y. \& Kutsal, T. 1996. The Selective Biosorption of Chromium (VI) and Copper (II) ions from binary metal mixtures by $R$. arrhizus. Process Biochemistry 31: 561-572.

Sag, Y. \& Kutsal, T. 1998. The simultaneous biosorption of $\mathrm{Cr}(\mathrm{VI}), \mathrm{Fe}$ (III) and Cu (II) on Rhizopus arrhizus. Process Biochemistry 33: 571-579.

Sag, Y. \& Kutsal, T. 2000. Determination of the biosorption heats of heavy metal ions on Zoogloea ramigera and Rhizopus arrhizus. Biochemical Engineering Journal 6: $145-151$.

Sag, Y.; Yalçuk, A. \& Kutsal, T. 2001. Use of a mathematical model for prediction of the performance of the simultaneous biosorption of $\mathrm{Cr}$ (VI) and $\mathrm{Fe}$ (III) on Rhizopus arrhizus in a semi-batch reactor. Hydrometallurgy 59: 77-87.

Schipper, M.A.A. 1984. A revision of the genus Rhizopus I. The Rhizopus stolonifer group and Rhizopus oryzae. Studies in Mycology 25: 1-34.

Schipper, M.A.A. 1990. On certain species of Mucor with a key to all accepted species. Studies in Mycology 25: 1-53.

Sherf, A.F. 1943. A method for maintaining Phytomonas sepedonica for long periods without trasnf. Phytopathology 33: 30-32.

Silva, G.A.; Maia, L.C.; Silva, F.S.B. \& Lima, P.C.F. 2001. Potencial de infectividade de fungos microrrízicos arbusculares oriundos de área de caatinga nativa e degradada por mineração, no Estado da Bahia, Brasil. Revista Brasileira de Botânica 24(2): 135-143.
Skugins, J. 1984. Microbial ecology of desert soils. Advances in Microbial Ecology 7: 49-91.

Souza, F.A. \& Silva, E.M.R. 1996. Micorrizas arbusculares na revegetação de áreas degradadas. Pp. 225-290. In: J.O. Siqueira (ed.). Avanços em Fundamentos e Aplicação de Micorrizas. Lavras, Universidade Federal de Lavras/ Departamento de Ciências do Solo e Departamento de Ciências Florestais.

Stamford, T.L.M.; Araujo, J.M. \& Stamford, M.P. 1998. Atividade enzimática de microrganismos isolados do jacatupé (Pachyrhizus erosus L. Urban). Ciência e Tecnologia de Alimentos 18(4): 382-385.

Tatsuyama, K.; Egawa, H.; Senmaru, H.; Yamamoto, H.; Ishioka, S.; Tamatsukuri, T. \& Saito, K. 1977. Cadmium and copper distributions in water and soil of $\mathrm{Mt}$. Homanzan and its neighborhood. Bulletin of the Faculty of Agriculture. Shimane University 11: 18-22.

Tatsuyama, K.; Egawa, H.; Yamamoto, H. \& Inoue, K. 1974. Influence of metallic compounds on soil microflora in farm polluted by chemicals. Transaction of the Mycological Society of Japan 15: 246-250.

Valsecchi, G.; Giglioti, C. \& Farini, A. 1995. Microbial biomass, activity and organic matter accumulation in soils contamined with heavy metals. Biology and Fertile Soils 20: $253-259$.

Vandame, E. \& Derycke, D. 1983. Microbial inulinases: fermentation process, properties and applications. Advances in Applied Microbiology 29: 139-176.

Volesky, B. 1994. Advances in biosorption of metals: Selection of biomass types. FEMS Microbiology Reviews 14(4): 291-302.

Warkup, J.H. 1950. The soil-plate method for isolation of fungi from soil. Nature 166: 117-150.

Yamamoto H.; Tatsuyama, K.; Egawa H. \& Furuta, T. 1981. Microflora in soils polluted by copper mine drainage. Japanese Journal of Soil Science and Plant Nutrition 54: 212-216.

Yamamoto, H.; Tatsuyama, K. \& Uchiwa, T. 1985 Fungal flora of soil polluted with copper. Soil Biology and Biochemistry 17(6): 785-790.

Zibilske, L.M. \& Wagner, G.H. 1982. Bacterial growth and fungal genera distribution in soil amended with sewage sludge containing cadmium, chromium and copper. Soil Science 134: 364-370. 